\title{
Population Structure in Cultivated Lettuce and Its Impact on Association Mapping
}

\author{
Ivan Simko ${ }^{1}$ \\ USDA-ARS, Crop Improvement and Protection Research Unit, 1636 East Alisal Street, Salinas, \\ CA 93905 \\ Jinguo Hu \\ USDA-ARS, Northern Crop Science Laboratory, 1307 North 18th Street, Fargo, ND 58105
}

\begin{abstract}
AdDitional IndeX words. Lactuca sativa, relative kinship, linkage disequilibrium
Abstract. The association mapping technique is a useful tool for detecting markers linked to the genes underlying the variation of a trait among elite cultivars. To avoid false-positive results due to unrecognized population structure in the analyzed set of individuals, the subpopulations need to be identified. Fifty-four lettuce (Lactuca sativa L.) cultivars representing five horticultural types important in North America, together with six accessions from two wild species (Lactuca saligna $\mathrm{L}$. and Lactuca serriola L.), were assayed for polymorphism with target region amplified polymorphism (TRAP) marker loci. The model-based clustering approach recognized three main subpopulations in cultivated lettuce that are well separated from wild species. Although the clustering based on molecular markers was generally in good agreement with horticultural types, some cultivars were classified differently or showed mixed origin. The effect of population structure on association mapping was tested on four traits with strong or weak correlation to the lettuce horticultural type and monogenic or polygenic mode of inheritance. Traits that were strongly correlated with lettuce types displayed many false-positive results when population structure was ignored, but the spurious associations disappeared when estimates of population structure or relative kinship (both based on molecular markers) were included in the statistical model. Using of horticultural types as covariate was not sufficient to control for spurious associations in the monogenic trait with strong correlation to lettuce types. The best approach to avoid spurious associations in lettuce association studies is to assess relatedness of accessions with molecular markers and to include this information into the statistical model.
\end{abstract}

The most common method for mapping genes in lettuce and other cultivated plant species is genetic linkage mapping. This approach involves generating populations derived from single crosses and estimating recombination frequencies between marker loci and the genes of interest. However, such mapping populations sample only a small proportion of all possible alleles. Moreover, to increase marker polymorphism, one of the parental genotypes is frequently derived from a wild species (or a primitive cultivar) that is often not representative of gene effects observed in elite cultivars.

In contrast to genetic linkage mapping, association mapping is a method that detects relationships between phenotypic variation and genetic polymorphisms in existing cultivars, without the need for developing new mapping populations. The method was originally applied in human genetics but has recently become a useful tool for mapping genes in plants. This linkage disequilibrium (LD)-based method effectively incorporates the effect of many past generations of recombination into a single analysis (Jorde, 2000). The association mapping technique, which provides the means for detecting genes underlying the variation of a trait among elite cultivars, is thus complementary to the linkage mapping method that effectively

Received for publication 27 Apr. 2007. Accepted for publication 20 Sept. 2007. This project was supported in part by the California Lettuce Research Board and the U.S. Department of Agriculture, Agricultural Research Service research programs.

The authors thank E.J. Ryder and M.D. Ospina-Giraldo for their critical review of the manuscript. Three anonymous reviewers made critical comments that helped to improve the manuscript.

${ }^{1}$ Corresponding author. E-mail: isimko@pw.ars.usda.gov. locates genes segregating in a population originating from two individuals (Simko, 2004). Early results achieved with association mapping in crops (Remington et al., 2001; Simko et al., 2004a, 2004b; Thornsberry et al., 2001; Wilson et al., 2004) indicate that this technique allows the identification of markers linked to genes and genomic regions associated with desirable traits in the elite material.

The main drawback of association mapping is a possibility of false-positive results due to an unrecognized population structure. Population structure is the presence of subpopulations in the sample in which individuals are more closely related to each other than the average pair of individuals taken at random in the population (Breseghello and Sorrells, 2006). When the trait of interest (e.g., a disease resistance) is more prevalent in one subpopulation than others, the trait will be associated with any marker allele that is in high frequency in that subpopulation (Ewens and Spielman, 1995; Lander and Schork, 1994; Pritchard and Rosenberg, 1999). Therefore, estimates of the population structure are prerequisite for successful implementation of the association mapping approach in admixed populations. Some authors avoided the problem of population structure by conducting statistical analyses within separate subpopulations (Garris et al., 2003; Simko et al., 2004a); however, this approach also reduces the statistical power of association mapping (Cardon and Palmer, 2003). To compute unbiased estimates of allele effects in structured populations, information about population structure needs to be included into the statistical model (Pritchard et al., 2000b).

At this time, no information is available on the distribution of alleles in subpopulations of lettuce. The objective of this research was to assess population structure and test the 
feasibility of association mapping analysis in cultivated lettuce. Although population structure assessment often relies upon a priori grouping on the basis of phenotypes (e.g., horticultural types in lettuce), this classification may not accurately describe the genetic structure of populations (Rosenberg et al., 2001). Thus, we used TRAP molecular markers to determine population structure (Pritchard and Rosenberg, 1999; Pritchard et al., 2000a, 2000b) among cultivars from five horticultural types. In addition to assessment of population structure, a relative kinship was calculated from marker data. The kinship coefficient is the probability that two homologous genes are identical by descent (Ritland, 1996; Yu et al., 2006). Similarly as assessment of population structure, relative kinship can be used to control for false-positive results in association studies. Subsequently, three approaches were compared for their efficiency to control spurious associations: 1) phenotypic classification into horticultural types, 2) assessment of population structure, and 3) estimate of relative kinship.

Association tests were performed between molecular markers and four phenotypic traits (lettuce dieback resistance, seed color, leaf margin undulation, and head height) that were selected for the analysis because of their different relationships to the lettuce horticultural types and different mode of inheritance. Our previous experience indicated that dieback resistance and head height are more type-related than seed color and leaf margin undulation. Dieback resistance is observed in modern icebergtype cultivars, while nearly all modern red leaf and romaine cultivars are highly susceptible. Resistance in iceberg lettuce is conferred by a dominant allele at a single locus (Grube et al., 2005). Information about the genetics of head height is limited, but romaine cultivars generally have greater head height. Leaf margin undulation is more profound in some leaf-type lettuces but is negligible in romaine, latin, and butterhead cultivars. Both the head height and the leaf margin undulation traits appear to be quantitatively inherited. Seed color is evenly distributed in all horticultural types. The inheritance of seed color is under the control of a single gene, with black color being dominant over white (Durst, 1930; Ryder, 1975).

\section{Materials and Methods}

Plant material. A set of 54 lettuce cultivars used for the population structure assessment represents five horticultural types cultivated in North America. The set includes 15 butterhead-type cultivars, 14 iceberg-type cultivars, 12 leaf-type cultivars, 12 romaine-type cultivars, and one latin-type cultivar. In addition to cultivated lettuce, three accessions each of two wild-type species Lactuca serriola and Lactuca saligna were also included in the analysis. The 60 accessions evaluated in this study are described in Table 1.

Plant phenotypic evaluation. Dieback resistance data (I. Simko, unpublished) were obtained from field observations as described in Grube et al. (2005). Susceptibility was evaluated by seeding lettuce directly in the field in Salinas, CA, from which lettuce necrotic stunt virus (LNSV) had previously been isolated from plants exhibiting characteristic dieback symptoms (Grube et al., 2005). The experiment comprised two complete blocks, with $\approx 30$ plants per genotype per block. Plants were seeded 1 Mar. 2006 in two rows on 1-m-wide beds and were thinned to obtain spacing of $30-\mathrm{cm}$ between plants. Standard commercial practices were used for irrigation, fertilization, and pest control. The percentage of plants that showed symptoms was recorded at harvest maturity and used for statistical analysis. Data for the other three traits were acquired from the Lettuce Cultivar Database (COMPOSITdb, 2007) and combined with our observations (I. Simko, unpublished). Leaf margin undulation and head height were rated on a scale from 1 to 9 , where higher numbers indicate a more undulated leaf margin and greater head height. Seed color was rated as white (0) or black (1).

Molecular MARKer GenOtYPING. All genotyping was performed with TRAP markers according to standardized protocol (Hu and Vick, 2003). Population structure was assessed with previously published TRAP marker data of $\mathrm{Hu}$ et al. (2005) combined with genotyping carried out on the cultivar Paragon (J. Hu, unpublished). Marker data were obtained from 18 primer pair combinations (all, but Lac08 of $\mathrm{Hu}$ et al., 2005), each consisting of a fixed primer and an arbitrary primer. The fixed primers were designed against selected EST sequences in the Compositae Genome Project Database (CGPDB, 2007) for surveying genetic variability in Compositae species. Each of the arbitrary primers consists of three parts: the selective nucleotides, the AT- or GC-rich "core" regions, and the filler sequences that make the $5^{\prime}$ end. The arbitrary primers were $5^{\prime}$ end-labeled with IR dye 700 or IR dye 800 for autodetection. More details of the procedure can be found in Hu et al. (2005). Polymorphic fragments were visually scored and classified as present (1) or absent (0).

Population STRUCTURE. Eighteen primer pair combinations yielded 388 polymorphic markers that were used to estimate population structure among 60 accessions. A subset of 107 markers polymorphic in cultivated lettuce was then used to infer population structure among cultivars only, while 281 markers monomorphic in L. sativa were excluded from this test. Both polymorphic and monomorphic markers originated from all tested primer pair combinations. To assign individuals into subpopulations we employed the dominant-marker model of the computer program Structure 2.2 (Falush et al., 2003; Pritchard et al., 2000a). The model also assumes that some fraction $\left(q_{k}\right)$ of each individual's genome originates from each of the $K$ subpopulations. Individuals are probabilistically assigned to the subpopulations in such a way as to achieve Hardy-Weinberg equilibrium for the loci within subpopulations. Although the individual accessions can belong to multiple subpopulations, the sum of $q$ values across all subpopulations for an individual is equal to $1\left(\sum_{k} q_{k}=1\right)$. Population analysis was performed with a predefined number of subpopulations $(K)$ ranging from 1 to 10 . Five runs were completed for each $K$, with 100,000 iterations, following a burn-in period of 20,000 iterations to find the optimal number of subpopulations and membership of each accession.

Pairwise clustering analysis was performed as in Rosenberg et al. (2001). Four horticultural types of cultivated lettuce (butterhead, iceberg, leaf, and romaine) plus two wild species were included in the analysis. The latin-type was not analyzed, as only a single accession belongs to this type. For each pairwise test, only two horticultural types (or species) were selected and the population structure was analyzed. In total, 15 pairwise tests were performed on four horticultural types and two wild species. All pairwise tests were carried out using 50,000 iterations, following a burn-in period of 20,000 iterations, with $K=2$. The clustering success rate was measured as the genome fraction of individual accessions assigned correctly to the predefined lettuce type (or species). 
Table 1. List of Lactuca accessions used in the study, their classification into horticultural types (or species), and estimated fraction $\left(q_{a}, q_{b}, q_{c}\right)$ of the accession's genome that originates from three inferred subpopulations (subpopulations A, B, and C). ${ }^{\mathrm{z}}$

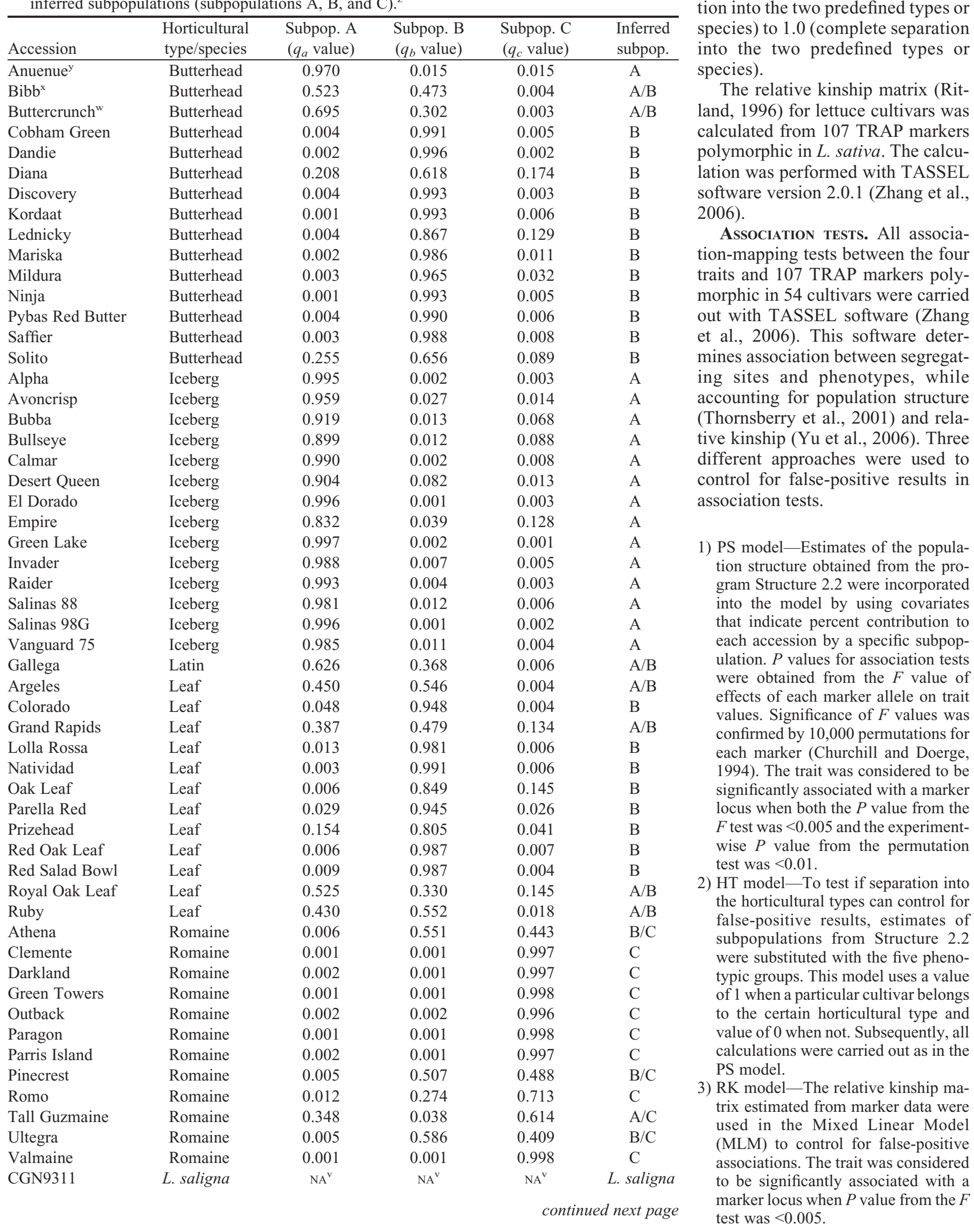

The values of clustering success rate can range from 0.5 (no separation into the two predefined types or species) to 1.0 (complete separation into the two predefined types or ecies).

The relative kinship matrix (Ritland, 1996) for lettuce cultivars was calculated from 107 TRAP markers lation was performed with TASSEL software version 2.0.1 (Zhang et al.,

tion-mapping tests between the four traits and 107 TRAP markers polymorphic in 54 cultivars were carried out with TASSEL software (Zhang . relative kinship (Yu et al., 2006). Three different approaches were used to control for false-positive results in

PS model-Estimates of the populaion structure obtained from the prointo the model by using covariates that indicate percent contribution to were obtained from the $F$ value of clues. Significance of $F$ values was 1994). The trait was considered to be $F$ test was $<0.005$ and the experimentwise $P$ value from the permutation

the horticultural types can control for false-positive results, estimates of ypic groups. This model uses a value of 1 when a particular cultivar belongs to the certain horticultural type and RK model-The relative kinship matrix estimated from marker data were (MLM) to control for false-positive associations. The trait was considered marker locus when $P$ value from the $F$ test was $<0.005$. ing sites and phenotypes, while 
Table 1. Continued.

\begin{tabular}{llcccc}
\hline Accession & $\begin{array}{c}\text { Horticultural } \\
\text { type/species }\end{array}$ & $\begin{array}{c}\text { Subpop. A } \\
\left(q_{a} \text { value }\right)\end{array}$ & $\begin{array}{c}\text { Subpop. B } \\
\left(q_{b} \text { value }\right)\end{array}$ & $\begin{array}{c}\text { Subpop. C } \\
\left(q_{c} \text { value }\right)\end{array}$ & $\begin{array}{c}\text { Inferred } \\
\text { subpop. }\end{array}$ \\
\hline PI 491204 & L. saligna & $\mathrm{NA}^{\mathrm{v}}$ & $\mathrm{NA}^{\mathrm{v}}$ & $\mathrm{NA}^{\mathrm{v}}$ & L. saligna \\
UC93US14 & L. saligna & $\mathrm{NA}^{\mathrm{v}}$ & $\mathrm{NA}^{\mathrm{v}}$ & $\mathrm{NA}^{\mathrm{v}}$ & L. saligna \\
94lact30-16 & L. serriola & $\mathrm{NA}^{\mathrm{u}}$ & $\mathrm{NA}^{\mathrm{u}}$ & $\mathrm{NA}^{\mathrm{u}}$ & L. serriola \\
94lact34-29 & L. serriola & $\mathrm{NA}^{\mathrm{u}}$ & $\mathrm{NA}^{\mathrm{u}}$ & $\mathrm{NA}^{\mathrm{u}}$ & L. serriola \\
W66336A & L. serriola & $\mathrm{NA}^{\mathrm{u}}$ & $\mathrm{NA}^{\mathrm{u}}$ & $\mathrm{NA}^{\mathrm{u}}$ & L. serriola \\
\hline
\end{tabular}

were $<0.5$, indicating overall high similarity between classifications based on horticultural type and the model-based clustering approach. Low $q$ values $(<0.5)$ were observed mostly because of grouping butterhead- and leaf-types together and because of placing the butterhead 'Anuenue' into the sub${ }^{\mathrm{z}}$ When an accession has $q>0.3$ for two subpopulations, the accession is assigned into mixed subpopulation $(\mathrm{A} / \mathrm{B}, \mathrm{A} / \mathrm{C}, \mathrm{B} / \mathrm{C})$.

${ }^{y}$ Center for Genetic Resources (CGR, 2007) classifies 'Anuenue' as iceberg-type lettuce.

${ }^{x}$ CGR (2007) classifies 'Bibb' as latin-type lettuce.

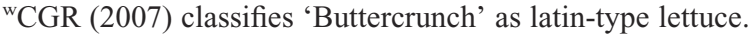

"The accession clusters into the $L$. saligna subpopulation.

"The accession clusters into the L. serriola subpopulation.

population comprising only icebergtype lettuces.

Allele Frequencies. To examine the difference in allele distribution among three Lactuca L. species, we compared frequency of alleles at 388 markers. Fisher's exact test revealed that 233 markers $(\approx 60 \%)$ have significantly $(P<0.01)$ differ-

A control test that ignores population structure was also included for comparison. All calculations were carried out as in the PS model, however, without covariates.

\section{Results}

Population STRuCture. Analysis of 60 accessions with 388 TRAP markers identified five distinct subpopulations (Fig. 1 Top). As expected, two of the subpopulations comprised only the L. serriola and L. saligna accessions. Fifty-four cultivars of L. sativa were divided into three subpopulations. The presence of three subpopulations in cultivated lettuce was confirmed with the additional test in which only markers polymorphic in L. sativa were included in the analysis (Fig. 1 Bottom). Subpopulation A contained 14 iceberg-type cultivars plus a single butterhead-type cultivar (Anuenue), subpopulation B contained a mixture of 12 butterhead and eight leaf-type cultivars, and subpopulation $\mathrm{C}$ consisted of eight romaine-type cultivars only. Eleven cultivars had a coefficient $q>0.3$ for two subpopulations and therefore were assigned into mixed subpopulations. Seven cultivars (two butterhead, one latin, and four leaf-type) belonged to subpopulation $\mathrm{A} / \mathrm{B}$, three romainetype cultivars belonged to subpopulation $\mathrm{B} / \mathrm{C}$, and a single romaine-type cultivar was classified as belonging to subpopulation $\mathrm{A} / \mathrm{C}$ (Table 1).

Pairwise Clustering. Four horticultural types (butterhead, iceberg, leaf, and romaine) and two wild species (L. serriola and L. saligna) allowed 15 pairwise clustering comparisons. All nine tests that included wild species had very high clustering success rate $(>0.990$, data not shown) without any misclassification between species, indicating a clear separation of accessions into the predefined subpopulations. Also, the clustering test that included a combination of iceberg-type and romainetype cultivars showed very high clustering success rate of 0.982 (Table 2). In contrast, the lowest clustering success rate of 0.683 and highest percentage of misclassified cultivars (43\%) was observed when only butterhead-type and leaf-type cultivars were used for the analysis. These observations confirm results from the study of population structure, which indicated that butterhead- and leaf-type cultivars form a single subpopulation, while iceberg and romaine lettuces are well separated from each other. When $q$ values for the predefined horticultural types were analyzed, $80 \%$ of them were $>0.9$. Only $12 \%$ of the $q$ values ent allele frequency in the three species. A similar comparison was also made among three inferred subpopulations within the L. sativa species. From 107 TRAP markers detected in lettuce cultivars, $60(\approx 56 \%)$ had a significantly different allele frequency.

Association TESTS. A test of associations between 107 TRAP markers polymorphic in L. sativa and four phenotypic traits detected significant marker-trait association for all traits using all three population-structure controlling models and the control. When population structure was not accounted for (control), 24 markers were significantly associated with the four horticultural traits. Ten of these markers were associated with dieback resistance, seven with head height, four with leaf margin undulation, and three with seed color. Using horticultural type (HT), population structure (PS), or relative kinship (RK) as covariates decreased the number of significant markers in almost all traits. In total, there were 11 significant associations detected when PS was used as covariate and 12 when HT or RK was used as covariate (Table 3 ).

The three models and the control usually detected the same markers when traits with no (or limited) correlation to horticultural type were tested. For example, the same three markers for seed color were detected in control, the PS model, and the RK model. One of these three markers was also detected when using the HT model. The situation was similar for leaf margin undulation. Three of the four markers significantly associated with the trait in the control test were also detected in the PS model. Two of these markers were then detected in the RK model, and one of these two markers was detected also in the HT model.

In contrast, dieback resistance and head height, which are strongly correlated with horticultural type, showed more significant associations. Moreover, markers detected with one model were often not detected by other models, as indicated by the number of unique markers for each test. While there was only a single unique marker for leaf margin undulation and none for seed color, association testing detected nine unique markers for head height and seven for dieback resistance.

\section{Discussion}

Population structure. Our test of population structure among 54 lettuce cultivars from five horticultural types identified three major subpopulations. Two of the subpopulations (A and $\mathrm{C}$ ) were formed from iceberg- and romaine-type cultivars 


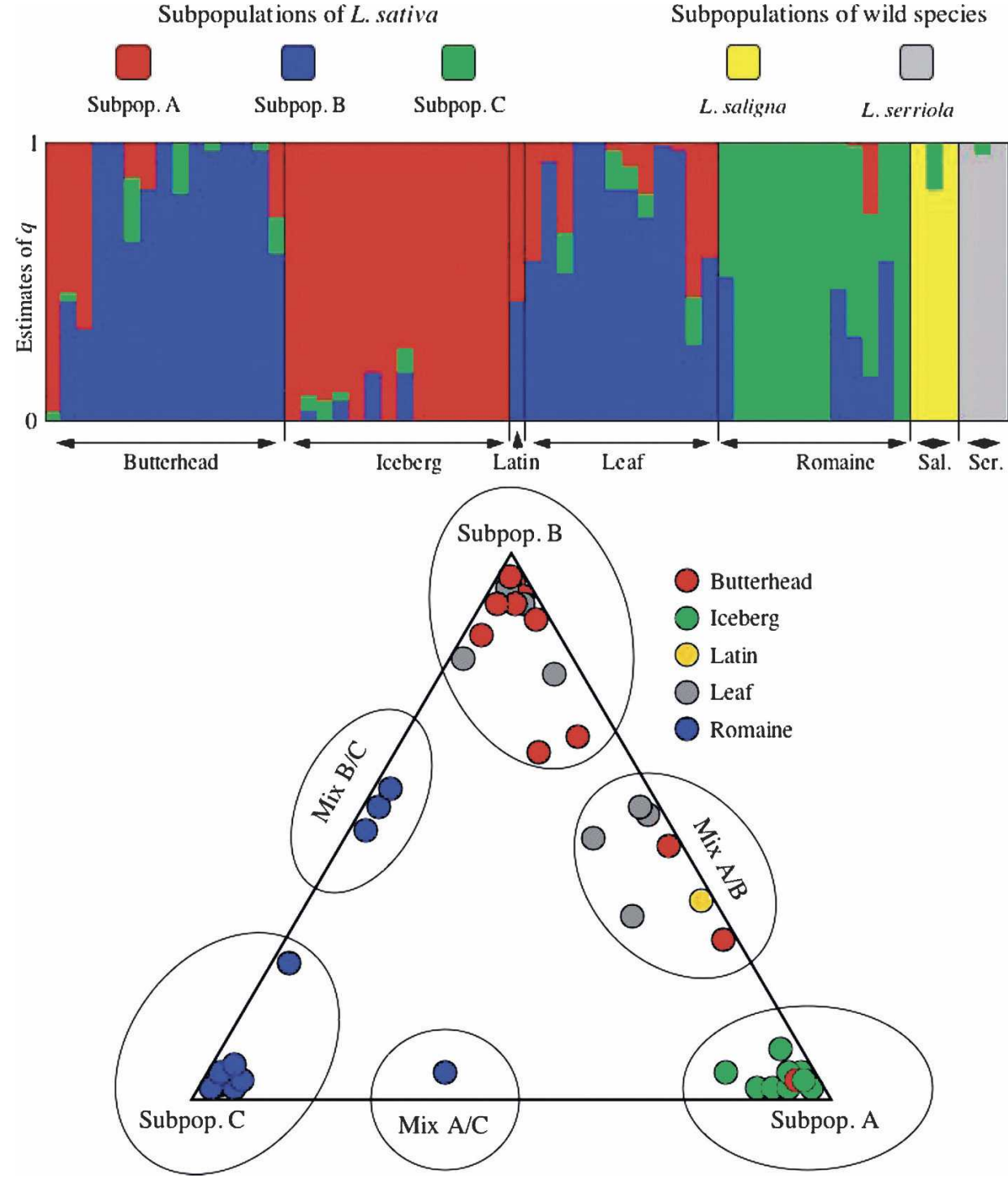

Fig. 1. (Top) Bar plot of population structure estimates for 60 Lactuca sativa, Lactuca saligna, and Lactuca serriola accessions. Population structure was assessed with 388 TRAP markers. Each accession is represented by a single vertical bar broken into five colored segments, with lengths proportional to $q$ of the five inferred subpopulations $(K=5)$. The sum of $q$ values for each bar is $1\left(\sum_{k} q_{k}=1\right)$. The horizontal axis shows the five horticultural types (butterhead, iceberg, latin, leaf, romaine) and two wild species (L. saligna, L. serriola). Accessions are in the same order as in Table 1. (Bottom) Triangle plot of population structure estimates for 54 cultivars with 107 TRAP markers that are polymorphic in L. sativa. A colored point represents each accession, with colors corresponding to the five horticultural types (butterhead, iceberg, latin, leaf, romaine). The estimated vector for an accession consists of three $q$ values that correspond to the three inferred subpopulations $(K=3)$. The sum of $q$ values for an accession is $1\left(\sum_{k} q_{k}=1\right)$. For an individual accession, the distance to one edge of the triangle gives each of the three $q$ values. Accessions that are in one of the corners are thus assigned completely to the corresponding subpopulation.

only, respectively. A single exception occurred when the butterhead-type cultivar Anuenue was assigned into subpopulation A with all iceberg-type cultivars (Fig. 1, Table 1). However, this butterhead lettuce resembles at maturity a small iceberg-type lettuce (COMPOSITdb, 2007) and is sometimes also classified as iceberg (Center for Genetic Resources, 2007). Butterhead- and leaf-type cultivars form the single subpopulation B. Clustering of these two horticultural types together was also confirmed by pairwise analysis that was not able to distinguish between butterhead and leaf types (Table 2).

Eleven cultivars were assigned into mixed subpopulations, indicating that some accessions cannot be easily classified and are a mixture of two subpopulations. However, it is likely that sampling more cultivars per horticultural type would improve clustering resolution. It was shown that the power to detect stratification increases with sample size, although larger numbers of molecular markers can also increase clustering accuracy to some extent (Cardon and Palmer, 2003; Long and Langley, 1999; Pritchard and Donnelly, 2001; Rosenberg et al., 2001).

Relative kinship coefficient is another approach that uses molecular marker data to assess cultivar relatedness. The coefficient grows when accessions are more closely related. For the lettuce sample, an Lshaped distribution was observed, with $60 \%$ of the pairwise kinship estimates equal to 0 and the remaining estimates distributed from 0.05 to 1.0 (data not shown). This distribution is highly similar to the one observed in a set of maize (Zea mays L.) accessions (Yu et al., 2006). The only noticeable difference between the two distributions was the higher fraction $(80 \%)$ of zero values in maize. Although there is not enough data to draw a reliable conclusion from this difference, it is likely that an overall kinship coefficient among 54 lettuce cultivars is higher than among 277 maize inbred lines.

Alternatively, the degree of relatedness among individuals and estimate of genetic variance components for association mapping can be derived from known pedigrees (Kennedy et al., 1992; Simko et al., 2004a). However, a pedigreebased estimate may be limited by available information, and the lack of adequate data for some genotypes renders them virtually useless for this type of analysis (Simko, 2004). It was suggested that molecular markers can be used to compensate for missing pedigree information (Hill et al., 1996). When the AFLP-based genetic distance estimates for the sample pairs of individuals were compared with those calculated from pedigree data, a linear regression indicated strong correlation between the assessments. However, the relationship deviated considerably from linearity when lettuce accessions were closely related. It was speculated that the difference may reflect the selection criteria used in the production of newly created lines (Hill et al., 1996). Thus pedigree information provides a useful guide; however, selection and genetic drift can cause considerable discrepancies between pedigree and genetic constitution (Liu et al., 2003). 
Table 2. Pairwise tests of clustering success rate with four horticultural types of lettuce. ${ }^{\mathrm{z}}$

\begin{tabular}{|c|c|c|}
\hline Pair of horticultural types ${ }^{y}$ & $\begin{array}{c}\text { Clustering success rate } \\
{[\text { mean } \pm \mathrm{SE}(0.5-1.0 \text { scale })]}\end{array}$ & $\begin{array}{l}\text { Incorrectly placed cultivars } \\
\qquad(\%)\end{array}$ \\
\hline Butterhead-iceberg & $0.890 \pm 0.046$ & 10.0 \\
\hline Butterhead-leaf & $0.683 \pm 0.075$ & 43.0 \\
\hline Butterhead-romaine & $0.880 \pm 0.043$ & 12.5 \\
\hline Iceberg-leaf & $0.895 \pm 0.034$ & 8.3 \\
\hline Iceberg-romaine & $0.982 \pm 0.012$ & 0.0 \\
\hline Leaf-romaine & $0.884 \pm 0.045$ & 12.5 \\
\hline
\end{tabular}

${ }^{\mathrm{z}}$ The clustering success rate was measured as the genome fraction of individual accessions assigned correctly to the predefined horticultural types (or species). Values can range from 0.5 (no separation into the two predefined types or species) to 1.0 (complete separation into the two predefined types or species). The "incorrectly placed cultivars" category identifies percent of cultivars [mean \% ( $0 \%$ to $50 \%$ scale)] that are placed in a different horticultural type than expected.

${ }^{\mathrm{y}}$ All pairwise clustering tests that include wild species have a success rate $>0.990$, and all accessions are placed in the expected horticultural type or species.

Yet another approach to avoid the problem with population structure is to perform association mapping analysis within individual subpopulations (Garris et al., 2003; Simko et al., 2004a). However, the reduction of false positives allowed by separate analysis of subpopulations comes at the cost of statistical power (Cardon and Palmer, 2003).

Association mapping. Considering that resistance to lettuce dieback is monogenic, finding 10 markers significantly associated with this trait in the association test without covariance (Table 3) indicates that many of the marker-trait associations are likely to be false positives. Using horticultural type as a covariate did not substantially decrease the number of significant associations compared with control. Moreover, six out of the nine markers detected in the HT model were identical to those identified in the control test. Although the exact position of these markers on the molecular linkage map is not known, preliminary linkage analysis (results are not shown) indicates that they originate from at least two distinct genomic regions. Incorporation of population structure (PS model) or relative kinship (RK model) into the statistical test decreased the number of significant markers to two (tightly linked, PS model) or one (RK model), respectively. Unfortunately, present data do not determine whether those significant markers are actually linked to the resistance gene or if the association is a false positive. In species such as maize, where LD decays rapidly [100-200 bp (Tenaillon et al., 2001); 1.5 kb (Remington et al., 2001)], few, if any, random markers are associated with a trait when marker density is similar to the present (Yu et al., 2006). Therefore all significant associations are considered to be false positives resulting from an unrecognized population structure. However, the situation may be different in species where LD is much longer. Limited data from the $D m 3$ resistance-gene region shows that LD in cultivated lettuce can reach several hundreds or even thousands of kilobases (Rouppe van der Voort et al., 2004). Such a long LD substantially increases the chance of detecting true association between a trait and random markers. In addition, $\approx 1 \%$ of TRAP markers used in the present study have sequences similar to protein kinase and homeobox genes ( $\mathrm{Hu}$ et al., 2005) that are involved in plant disease resistance and organ development processes, respectively. Therefore, it is possible that the significant associations detected with PS and RK models identify the true location of the dieback resistance gene.

Results from the seed color data are different from the dieback resistance results, indicating that population structure does not affect marker-trait associations in these two traits to the same extent. Three identical markers were detected in the control, PS, and RK model tests. One of those three markers was also detected in the HT model test, indicating a very good match between the four analyses. Detection of the same marker-trait associations is likely due to a similar distribution of seed color in all horticultural types. However, similar to dieback resistance, it is not possible to determine with certainty whether the three markers (that are in LD) identify a true genomic location of the seed color gene.

Two other traits - head height and leaf margin undulationshow distributions indicating effects of multiple genes. However, again, the trait that correlates strongly with horticultural type (greater head height in romaine-type cultivars) displayed more marker-trait associations. Conversely, results from leaf margin undulation, the trait that is weakly correlated with horticultural type, resemble the pattern seen in seed color. Similarly to the tests with seed color, the low number of significant associations was accompanied by very few unique associations (a single association in control), demonstrating good correlation among the four tests. These observations agree with the principle that prevalence of a trait in one subpopulation leads to false-positive association with alleles that are in high frequency in that

Table 3. Number of markers significantly associated with the four traits in the set of 54 lettuce cultivars. ${ }^{z}$

\begin{tabular}{|c|c|c|c|c|c|c|}
\hline \multirow[b]{2}{*}{ Trait } & \multirow[b]{2}{*}{ Inheritance } & \multirow{2}{*}{$\begin{array}{l}\text { Correlation to } \\
\text { horticultural } \\
\text { type }\end{array}$} & \multicolumn{4}{|c|}{ Significant markers [no. (no. of unique markers for the test)] } \\
\hline & & & $\begin{array}{l}\text { Control-no } \\
\text { covariance }\end{array}$ & $\begin{array}{c}\text { Covariance test-HT } \\
\text { model }\end{array}$ & $\begin{array}{c}\text { Covariance test-PS } \\
\text { model } \\
\end{array}$ & $\begin{array}{c}\text { Covariance test-RK } \\
\text { model } \\
\end{array}$ \\
\hline Dieback resistance & Monogenic & Strong & $10(3)$ & $9(3)$ & $2(1)$ & $1(0)$ \\
\hline Seed color & Monogenic & Weak & $3(0)$ & $1(0)$ & $3(0)$ & $3(0)$ \\
\hline $\begin{array}{l}\text { Leaf margin } \\
\text { undulation }\end{array}$ & Polygenic & Weak & $4(1)$ & $1(0)$ & $3(0)$ & $2(0)$ \\
\hline
\end{tabular}

${ }^{\mathrm{z}}$ For each trait the mode of inheritance (polygenic or monogenic) and correlation to horticultural type (strong or weak) is indicated, together with the number of significant markers (unique markers for the particular test are in parenthesis) detected for the trait with individual tests. Four tests were performed on each trait - a control test that ignores population structure, and three tests that use covariates to control for structured population: HT model uses horticultural type, PS model uses population structure, and RK model uses relative kinship. Estimates of both population structure and relative kinship were calculated from 107 TRAP markers polymorphic in lettuce cultivars. 
subpopulation (Pritchard and Rosenberg, 1999). This principle was observed in our tests regardless of the inheritance mode; there were 32 significant markers (seven of them unique) detected for the two polygenic traits, and 27 significant markers (10 of them unique) were detected for the two monogenic traits. The similarity in the number of significant markers for polygenic and monogenic traits illustrates that inheritance of a trait does not have a substantial effect on association tests.

Our results are in line with the observations on maize (Thornsberry et al., 2001; Wilson et al., 2004) and arabidopsis [Arabidopsis thaliana (L.) Heynh.] (Aranzana et al., 2005), where estimates of population structure substantially reduced the number of false positives. For even better separation of true from false-positive associations, Aranzana et al. (2005) suggested development of statistical methods that simultaneously infer the structure and the marker-trait associations.

MARKER TYPE. TRAP markers are dominant markers with high $(98 \%)$ reproducibility that can provide a powerful technique for fingerprinting lettuce cultivars (Hu et al., 2005). Analysis of marker distribution indicated that TRAP markers are well spread across the lettuce genome with a few clusters, each with two to five tightly linked markers ( $\mathrm{Hu}$ et al., 2005). Although all genotypic work in the present study was done with TRAP markers, we investigated whether other types of markers would yield comparable estimates of population structure. The results from fingerprinting with TRAP (Hu et al., 2005), AFLP (Hill et al., 1996), RFLP (Kesseli et al., 1991), SAMPL (Witsenboer et al., 1997), and isozyme (Kesseli and Michelmore, 1986) markers were examined and Dice's similarity coefficient (Dice, 1945; Nei and Li, 1979) for pairs of lettuce cultivars genotyped in multiple studies was approximated. The similarity coefficients were then recorded into the data matrix and compared. The correlation between matrices for TRAP markers and AFLP markers was 0.909, TRAP and SAMPL markers 0.610 , TRAP and RFLP markers 0.582 , and TRAP and isozyme markers 0.536 . All correlations, with the exception of the one that included isozymes, were significant at $P<0.01$ when evaluated with the Mantel test of correlation (Mantel, 1967) between two matrices. However, the AFLP markers showed results most similar to those obtained with TRAP markers and thus would likely yield the same or very similar population structure estimates. This outcome is not surprising, because both AFLP and TRAP markers show high resolution and similar results when used for lettuce cultivar fingerprinting (Hill et al., 1996; Hu et al., 2005; van Eeuwijk and Law, 2004).

\section{Conclusion}

Testing the set of 54 cultivars showed presence of subpopulations that were relatively similar, but not identical, with the classification based on horticultural type. We have observed that traits that are strongly correlated with horticultural type display many false-positive results when population structure is ignored; but the association is much less evident when estimates of population structure or relative kinship are included in the statistical model.

However, using of horticultural types as covariates (HT model) does not control well for spurious associations (e.g., dieback resistance). The reason is that phenotypic classification into horticultural types differs to some extent from genotypic relatedness. For example, molecular markers indicate that the cultivar Anuenue is highly similar to iceberg-type lettuces, although it is classified as butterhead. Similarly, butterhead cultivars Bibb and Buttercrunch are molecularly more similar to some latin cultivars (Gallega) or leaf-type cultivars (Argeles, Grand Rapids, Royal Oak Leaf, Ruby) than to other butterhead cultivars. Another reason why the HT model does not control well for spurious associations is the presence of subpopulations within horticultural type. For instance, three romaine cultivars (Athena, Pinecrest, Ultegra) form a distinct subpopulation that is molecularly between the "true" romaine cultivars and those in the group consisting from butterhead- and leaf-type lettuces. We do not have the complete pedigree information to compare how well our observations correspond to the pedigree data; however, previous work with AFLP markers shows that correlation between pedigree-based and marker-based similarity in lettuce accessions is strong for most, but very closely related individuals (Hill et al., 1996).

The PS and RK models show similar effectiveness in controlling spurious associations in three out of the four traits. However, the difference between the two models was observed in head height, the trait with polygenic inheritance and strong correlation to horticultural type. The PS model detected three significant associations (all of them unique), and the RK model detected six significant associations - two unique and four identical to those in the control. On the basis of our present results only, it is not possible to single out which of the significant markers are true positives and which are spurious associations due to structured population. However, assuming that most, if not all, significant associations detected in the control test are false positives, detection of four identical markers in the RK model indicates that these associations might also be false positives. These results are somewhat different from the previous comparison of the two models. When maize flowering time, ear height, and ear diameter were analyzed on a panel of 277 inbred lines, correction by the RK model was always better than the PS model (Yu et al., 2006). We speculate that dissimilarity between the two studies might be caused by population structure, extent of LD, or distribution of the analyzed traits.

In this study, we found that lettuce cultivars from five horticultural types form three distinct subpopulations. Although there was generally good agreement between a priori grouping on the basis of phenotypes and inferred population structure, simple phenotypic classification did not control well for false-positive results in the monogenic trait with strong correlation to horticultural type. Therefore, the best approach to avoid spurious associations in lettuce association studies is to assess relatedness of accessions with molecular markers and to include this information into the statistical model.

\section{Literature Cited}

Aranzana, M.J., S. Kim, K. Zhao, E. Bakker, M. Horton, K. Jakob, C. Lister, J. Molitor, C. Shindo, C. Tang, C. Toomajian, B. Traw, H. Zheng, J. Bergelson, C. Dean, P. Marjoram, and M. Nordborg. 2005. Genome-wide association mapping in arabidopsis identifies previously known flowering time and pathogen resistance genes. PLoS Genet. 1:531-539.

Breseghello, F. and M.E. Sorrells. 2006. Association analysis as a strategy for improvement of quantitative traits in plants. Crop Sci. 46:1323-1330.

Cardon, L.R. and L.J. Palmer. 2003. Population stratification and spurious allelic association. Lancet 361:598-604. 
Centre for Genetic Resources (CGR). 2007. Lettuce. 14 Nov. 2006 $<$ http://www.cgn.wur.nl/UK/CGN+Plant+Genetic+Resources/ Search + and + order + germplasm/On+line + search/Default.htm?page $=$ zoekgewas\&Cropnumber $=06>$.

Compositae Genome Project Database. 2007. Compositae genome project database: sequence retrieval. 10 Dec. 2003. <http://cgpdb. ucdavis.edu/database/seq_fasta_info.php $>$.

Churchill, G.A. and R.W. Doerge. 1994. Empirical threshold values for quantitative trait mapping. Genetics 138:963-971.

COMPOSITdb. 2007. Compositae species database: lettuce cultivar database. 21 Mar. 2007. <http://compositdb.ucdavis.edu/database/ lettcv2/display/>.

Dice, L.R. 1945. Measures of the amount of ecologic association between species. Ecology 26:297-302.

Durst, C.E. 1930. Inheritance in lettuce. Univ. Illinois Agr. Expt. Sta. Bul. 356:238-341.

Ewens, W.J. and R.S. Spielman. 1995. The transmission/disequilibrium test: history, subdivision, and admixture. Amer. J. Hum. Genet. 57: 455-464.

Falush, D., M. Stephens, and J.K. Pritchard. 2003. Inference of population structure using multilocus genotype data: linked loci and correlated allele frequencies. Genetics 164:1567-1587.

Garris, A.J., S.R. McCouch, and S. Kresovich. 2003. Population structure and its effect on haplotype diversity and linkage disequilibrium surrounding the $x a 5$ locus of rice (Oryza sativa L.). Genetics 165:759-769.

Grube, R.C., W.M. Wintermantel, P. Hand, R. Aburomia, D.A.C. Pink, and E.J. Ryder. 2005. Genetic analysis and mapping of resistance to lettuce dieback: a soilborne disease caused by tombusviruses. Theor. Appl. Genet. 110:259-268.

Hill, M., H. Witsenboer, M. Zabeau, P. Vos, R. Kesseli, and R. Michelmore. 1996. PCR-based fingerprinting using AFLPs as a tool for studying genetic relationships in Lactuca spp. Theor. Appl. Genet. 93: 1202-1210.

Hu, J. and B.A. Vick. 2003. Target region amplification polymorphism: a novel marker technique for plant genotyping. Plant Mol. Biol. Rptr. 21:289-294.

Hu, J., O.E. Ochoa, M.J. Truco, and B.A. Vick. 2005. Application of the TRAP technique to lettuce (Lactuca sativa L.) genotyping. Euphytica 144:225-235.

Jorde, L.B. 2000. Linkage disequilibrium and the search for complex disease genes. Genome Res. 10:1435-1444.

Kennedy, B.W., M. Quinton, and J.A. van Arendonk. 1992. Estimation of effects of single genes on quantitative traits. J. Anim. Sci. 70:2000-2012.

Kesseli, R., O. Ochoa, and R. Michelmore. 1991. Variation at RFLP loci in Lactuca spp. and origin of cultivated lettuce (L. sativa). Genome 34:430-436.

Kesseli, R.V. and R.W. Michelmore. 1986. Genetic variation and phylogenies detected from isozyme markers in species of Lactuca. J. Hered. 77:324-331.

Lander, E.S. and N.J. Schork. 1994. Genetic dissection of complex traits. Science 265:2037-2048.

Liu, K., M. Goodman, S. Muse, J.S. Smith, E. Buckler, and J. Doebley. 2003. Genetic structure and diversity among maize inbred lines as inferred from DNA microsatellites. Genetics 165:2117-2128.

Long, A.D. and C.H. Langley. 1999. The power of association studies to detect the contribution of candidate genetic loci to variation in complex traits. Genome Res. 9:720-731.

Mantel, N. 1967. The detection of disease clustering and a generalized regression approach. Cancer Res. 27:209-220.

Nei, M. and W.H. Li. 1979. Mathematical model for studying genetic variation in terms of restriction endonucleases. Proc. Natl. Acad. Sci. USA 76:5269-5273.

Pritchard, J.K. and N.A. Rosenberg. 1999. Use of unlinked genetic markers to detect population stratification in association studies. Amer. J. Hum. Genet. 65:220-228.
Pritchard, J.K. and P. Donnelly. 2001. Case-control studies of association in structured or admixed populations. Theor. Popul. Biol. 60:227-237.

Pritchard, J.K., M. Stephens, and P. Donnelly. 2000a. Inference of population structure using multilocus genotype data. Genetics 155:945-959.

Pritchard, J.K., M. Stephens, N.A. Rosenberg, and P. Donnelly. 2000b. Association mapping in structured populations. Amer. J. Hum. Genet. 67:170-181.

Remington, D.L., J.M. Thornsberry, Y. Matsuoka, L.M. Wilson, S.R. Whitt, J. Doebley, S. Kresovich, M.M. Goodman, and E.S. Buckler, IV. 2001. Structure of linkage disequilibrium and phenotypic associations in the maize genome. Proc. Natl. Acad. Sci. USA 98:11479-11484.

Ritland, K. 1996. Estimators for pairwise relatedness and individual inbreeding coefficients. Genet. Res. 67:175-185.

Rosenberg, N.A., T. Burke, K. Elo, M.W. Feldman, P.J. Freidlin, M.A.M. Groenen, J. Hillel, A. Mäki-Tanila, M. Tixier-Boichard, A. Vignal, K. Wimmers, and S. Weigend. 2001. Empirical evaluation of genetic clustering methods using multilocus genotypes from 20 chicken breeds. Genetics 159:699-713.

Rouppe van der Voort, J., A. Sorensen, D. Lensink, M. Van der Meulen, R. Michelmore, and J. Peleman. 2004. Decay of linkage disequilibrium in the $\mathrm{Dm} 3$ resistance-gene cluster of lettuce. Plant and Animal Genomes XII Conf., San Diego, CA, 10-14 Jan. 2004. Abstract no. P748.

Ryder, E.J. 1975. Linkage and inheritance in lettuce (Lactuca sativa L.). J. Amer. Soc. Hort. Sci. 100:346-349.

Simko, I. 2004. One potato, two potato: haplotype association mapping in autotetraploids. Trends Plant Sci. 9:441-448.

Simko, I., S. Costanzo, K.G. Haynes, B.J. Christ, and R.W. Jones. 2004a. Linkage disequilibrium mapping of a Verticillium dahliae resistance quantitative trait locus in tetraploid potato (Solanum tuberosum) through a candidate gene approach. Theor. Appl. Genet. 108:217-224

Simko, I., K.G. Haynes, E.E. Ewing, S. Costanzo, B.J. Christ, and R.W. Jones. 2004b. Mapping genes for resistance to Verticillium albo-atrum in tetraploid and diploid potato populations using haplotype association tests and genetic linkage analysis. Mol. Genet. Genomics 271:522-531.

Tenaillon, M.I., M.C. Sawkins, A.D. Long, R.L. Gaut, J.F. Doebley, and B.S. Gaut. 2001. Patterns of DNA sequence polymorphism along chromosome 1 of maize (Zea mays ssp. mays L.). Proc. Natl. Acad. Sci. USA 98:9161-9166.

Thornsberry, J.M., M.M. Goodman, J. Doebley, S. Kresovich, D. Nielsen, and E.S. Buckler, IV. 2001. Dwarf8 polymorphisms associate with variation in flowering time. Nat. Genet. 28:286-289. van Eeuwijk, F.A. and J.R. Law. 2004. Statistical aspects of essential derivation, with illustrations based on lettuce and barley. Euphytica 137:129-137

Wilson, L.M., S.R. Whitt, A.M. Ibanez, T.R. Rocheford, M.M. Goodman, and E.S. Buckler, IV. 2004. Dissection of maize kernel composition and starch production by candidate gene association. Plant Cell 16:2719-2733.

Witsenboer, H., J. Vogel, and R.W. Michelmore. 1997. Identification, genetic localization, and allelic diversity of selectively amplified microsatellite polymorphic loci in lettuce and wild relatives (Lactuca spp.). Genome 40:923-936.

Yu, J., G. Pressoir, W.H. Briggs, I.V. Bi, M. Yamasaki, J.F. Doebley, M.D. McMullen, B.S. Gaut, D.M. Nielsen, J.B. Holland, S. Kresovich, and E.S. Buckler. 2006. A unified mixed-model method for association mapping that accounts for multiple levels of relatedness. Nat. Genet. 38:203-208.

Zhang, Z., P.J. Bradbury, D.E. Kroon, T.M. Casstevens, and E.S. Buckler. 2006. TASSEL 2.0: a software package for association and diversity analyses in plants and animals. Plant and Animal Genomes XIV Conf., San Diego, CA, 14-18 Jan. 2006. Abstract no. P956/CP012. 